\title{
POSITIVE LINEAR OPERATORS CONTINUOUS FOR STRICT TOPOLOGIES
}

\section{J. A. CRENSHAW}

ABSTRACT. If $A$ is an $S W$ algebra of real-valued functions on a set $X$ equipped with the weak topology for $A$, and if $A$ separates its zero sets, then many results valued for $C^{b}(X)$ equipped with a strict topology remain true when $A$ is equipped with a strict topology. The concepts of a-additivity and tight positive linear operators are introduced. It is shown that if $T$ is a positive linear map on $A$ into $z$-separating $S W$ algebra $B$ and if $T\left(1_{A}\right)=1_{B}$, then there exists a continuous function $\phi$ on $Y$ (the domain of elements in $B$ ) into $X$ such that $T f(y)=f(\phi(y))$ if and only if $T$ is an algebraic homomorphism and $\tau$-additive.

Introduction. Let $X$ be a completely regular Hausdorff space. An algebra $A$ of real-valued functions on $X$ is an $S W$ algebra if $A$ separates the points of $X$, contains the constants and is uniformly closed. If $A$ is an $S W$ algebra which separates its zero sets, then Kirk [4] has shown that $A^{*}$ can be represented as the space $M(z(A))$ of finitely additive set functions on the Baire sets of $X$. Let $X_{A}$ be the extreme points of positive face in the unit ball in $A^{*}$ equipped with $\sigma\left(A^{*}, A\right)$. Let $\tau_{A}$ be the relative $\sigma\left(A^{*}, A\right)$ topology on $X$ embedded into $X_{A}$. Then $X_{A}$ is the compactification of $\left(X, \tau_{A}\right)$ for which $f \in C^{b}\left(X, \tau_{A}\right)$ has a continuous extension iff $f \in A$. Using Kirk's characterization and the techniques of Sentilles [9] many results on the strict topologies on $C^{b}(X)$ carry over to $A$.

If $A$ and $B$ are two $z$-separating $S W$ algebras defined on $X, Y$ respectively and are equipped with a strict topology $\beta_{\alpha}$, then continuous positive linear maps can be characterized in terms of additivity conditions. Also if $C=\left\{T \in L(A, B): \theta \leq T, T\left(1_{A}\right)=1_{B}\right\}$ then F. F. Bonsall, J. Lindenstrauss, and R. R. Phelps [1] have shown that the extreme points of $C$ are the alge-

Received by the editors September 24, 1973.

AMS (MOS) subject classifications (1970). Primary 46E25, 47B55; Secondary $28 \mathrm{~A} 40$.

Key words and phrases. Algebras of functions, strict topologies, positive linear operator, $\sigma$-additive, $\tau$-additive, tight, extreme linear operator, algebraic homomorphism. 
braic homomorphisms, and recently J. Hoffman-J $\varnothing$ rgensen [3] has shown that when $A=C^{b}(X)$ and $B=C^{b}(Y)$ then the algebraic homomorphisms which are $\beta_{0}$ continuous have a continuous map $\phi$ on $Y$ into $X$ such that $\operatorname{Tf}(y)=$ $f(\phi(y))$. We shall show in a different manner that such a $\phi$ exists for $T \epsilon$ $C$ iff $T$ is an extreme point of $C$ and $r$-additive.

1. Basic definitions and preliminaries. An $S W$ algebra $A$ is algebraically, topologically, and lattice isomorphic to $C\left(X_{A}\right)$ under $f \rightarrow \bar{f}$ for $\bar{f}$ the unique continuous extension of $f \in A$ from $X$ to $X_{A}$. Also $A$ is $z$-separating if for $Z_{1}, Z_{2}$ disjoint zero sets in $X$, there is an $f \in A$ with $f\left(Z_{1}\right)=$ $0 \leq f \leq 1=f\left(Z_{2}\right)$

For $\alpha$ an infinite cardinal, we define

$$
L_{\alpha}=\left\{Q \subset X_{A} \backslash X: Q=\bigcap_{\gamma \in I} \bar{Z}_{\gamma} \text {, card } I \leq \alpha, Z_{\gamma} \text { is a zero set in } X\right\} \text {. }
$$

Let $\tau$ be the least cardinal with the property that every closed set in $X$ is the intersection of at most $\tau$ zero sets. Since the zero sets $z(A)$ of $A$ form a basis for the $\tau_{A}$-closed sets, $\tau \leq$ card $2^{X}$ exists. For each $Q \in L_{\alpha}$, let $\beta_{Q}$ be the Hausdorff locally convex topology on $A$ generated by the seminorms $\left\{p_{g}: \bar{g}(Q)=0, p_{g}(f)=\|f \cdot g\|, f, g \in A\right\}$. Let $\beta_{a}$ denote the inductive limit topology on $A$ of the $B_{Q}$ topologies as $Q$ varies over $L_{a}$. We shall use F.D. Sentilles' notation for $\beta_{0}$ as the strongest locally convex topology on $A$ which agrees with the compact-open topology on norm bounded subsets of $A$. We note that $\beta_{a} \geq \beta_{a^{\prime}}$ if $\alpha \leq \alpha^{\prime}$, and $\beta_{0} \leq \beta_{a}$ for all $\alpha$ follows as in $[9,2.1]$.

Let $A, B$ be $z$-separating $S W$ algebras on $X, Y$ respectively, then $T \epsilon$ $L^{+}(A, B)$ if $T$ is a positive linear map on $A$ into $B$.

Definitions. Let $T \in L^{+}(A, B)$ and $m \in M^{+}(z(A))$ and card $I \leq \alpha$, then

(1) $T$ is a-additive if $T f_{\gamma} \downarrow \theta$ whenever $f_{\gamma} \downarrow \theta$ in $A$ for $\gamma \in I$.

(2) $m$ is $\alpha$-additive if $m\left(Z_{\gamma}\right) \rightarrow 0$ whenever $Z_{\gamma} \downarrow \varnothing, Z_{\gamma} \in z(A), \gamma \in I$.

(3) $T$ is tight if $\left\{T f_{i}\right\}$ is norm bounded and uniformly convergent to $\theta$ on compact subsets of $Y$ whenever $\left\{f_{i}\right\}$ has these properties in $A$.

The $\alpha$-additive (respectively tight) elements of $M(z(A))$ generate a band denoted by $M_{a}$ (respectively $M_{t}$ ). We remark that $\alpha$-additivity will be extensively examined in a more general setting in [5].

2. Some results for $C^{b}(X)$ valid for $A$. We note that for $Q \in L_{a}, C\left(X_{A} \backslash Q\right)$ is a Banach algebra with approximate identity $\left\{E_{\lambda}\right\}_{\lambda \in \boldsymbol{\Lambda}}$ with $\Lambda \leq \alpha$ (see [4, 1.5]). Also as shown in $[4, \$ 6], \mu \in M(z(A))$ has a unique extension $\bar{\mu}$ to the 
Baire sets on $X_{A}$, which in turn has a unique extension to a Borel measure $\bar{\nu}$ on $X_{A}$. With these remarks and 5.5 of [4] we can essentially repeat the standard arguments to establish the results below.

1. Proposition. $A\left(\beta_{\alpha}\right)$ and $A\left(\beta_{0}\right)$ are Hausdorff locally convex lattices.

Proof. For $\beta_{0}$ the result is clear since the compact-open topology is solid. For $\beta_{a}$ see [7, Theorem 2.2].

2. Proposition. $\beta_{a}, \beta_{0}$ are the finest locally convex topologies agreeing with their respective selves on each $r U$ for $r>0$ and $U$ the unit ball of $A$. The continuity of linear maps for each topology is determined on sets $r U$.

Proof. Use the remarks above and the arguments given by Sentilles culminating with $[9,4.1]$.

3. Proposition. $\beta_{a}$ is the topology of uniform convergence on the weak ${ }^{*}$ compact subsets of $M_{a}^{+}(z(A))$.

Proof. See the argument given by Mosiman in [7, 2.7].

4. Proposition. The following are equivalent for $\theta \leq \phi \in A(\|\cdot\|)^{*}$ :

(a) $\phi$ is a-additive.

(b) $\mu$ is a-additive, where $\mu \in M(z(A))$ represents $\phi$.

(c) $\bar{\mu}(Q)=0$ for all $Q \in L_{a}$.

(d) $\phi$ is $\beta_{a}$ continuous.

Proof. The proof uses the remarks above and follows the arguments given in $[10,24$, p. 174], $[6,2.4]$, and $[9,4.3]$.

5. Proposition. (a) $A\left(\beta_{0}\right)^{*}=M_{t}$.

(b) $S \subset M_{t}$ is $\beta_{0}$ equicontinuous iff $S$ is tight (norm bounded and for $\epsilon>0$ there is a compact $K \subset X$ with $|\mu|(X \backslash K)<\epsilon)$.

(c) $\beta_{0}$ is the topology of uniform convergence on the tight subsets of $M_{t^{*}}$

Proof. The arguments are those used by Sentilles in 4.3 and 5.1 of [9] where $\mu$ is the compact-regular Borel extension of $\mu \in M_{t^{*}}$. For the existence of such a unique extension of $\mu$ see, for example, $[5,2.12]$.

6. Proposition. Let $\delta_{p}$ be a point functional for $p \in X_{A}$. Then $p \in X$ iff $p \in M_{\tau}$ iff $p \in M_{t^{*}}$

Proof. If $p \in X$, then $\delta_{p}$ is tight so $r$-additive. If $p$ is not in $X$, then 
$\{f \in A: \bar{f}(p)=1\}$ forms a net $\left\{f_{\gamma}\right\}$ with $f_{\gamma} \downarrow \theta$ but $\delta_{p}\left(f_{\gamma}\right)=1$ so $\delta_{p} \notin M_{t}$.

3. Positive linear maps. In this section we shall assume $A, B$ are $z$ separating $S W$ algebras on $\left(X, \tau_{A}\right)$ and $\left(Y, \tau_{B}\right)$ respectively with compactifications $X_{A}$ and $Y_{B}$. We note that $T \in L^{+}(A, B)$ is norm continuous so that $T^{*}$ is a weak ${ }^{*}$ continuous linear map on $M(z(B))$ into $M(z(A))$.

7. Proposition. Let $T \in L^{+}(A, B)$ and let $\alpha \leq \alpha^{\prime}$. Then the following are equivalent.

(a) $T$ is $\beta_{a}-\beta_{a}$, continuous.

(b) $T^{*}\left(M_{a^{\prime}}(z(B))\right) \subset M_{a^{\prime}}(z(A))$.

(c) $T$ is a-additive.

Proof. (a) $\Rightarrow($ b). This follows from 4 .

(b) $\Leftrightarrow$ (c). Use 6 and $M_{t} \subset M_{a^{\prime}}$.

(b) $\Rightarrow$ (a). Let $W$ be a $\beta_{\alpha^{\prime}}$ neighborhood of $\theta$ in $B$. Then by 3 , there is a weak ${ }^{*}$ compact subset $D$ of $M_{a}^{+}(z(B))$ such that $D^{\circ} \subset W$. Since $T^{*}$ is weak ${ }^{*}$ continuous and positive, it follows from 3 that $\left(T^{*} D\right)^{\circ}=V$ is a $\beta_{a}$ neighborhood of $\theta$ in $A$. Clearly $T(V) \subset W$.

8. Proposition. The following are equivalent for $T \in L^{+}(A, B)$ :

(a) $T$ is tight.

(b) $T$ is $\beta_{0}-\beta_{0}$ continuous.

(c) $T^{*}$ maps tight subsets of $M(z(B))$ into tight subsets of $M(z(A))$.

Proof. $(a) \Leftrightarrow(b)$. Since the norm bounded nets which $\beta_{0}$ converge to $\theta$ in $A$ are the norm bounded nets which converge uniformly to $\theta$ on compact sets, the equivalence of (a) and (b) follows from the last statement in 2.

(b) $\Leftrightarrow(c)$. This follows from 5 since $M_{t}(z(B))$ and $M_{t}(z(A))$ are the dual spaces of $B\left(\beta_{0}\right)$ and $A\left(\beta_{0}\right)$ respectively and since the topology $\beta_{0}$ is the topology of uniform convergence on the tight subsets of $M_{t^{*}}$.

We remark that clearly $T$ is $\beta_{0}-\beta_{\alpha}$ continuous $\Rightarrow T$ is $\beta_{0}-\beta_{0}$ continuous $\Rightarrow T$ is $\beta_{a}-\beta_{0}$ continuous. However neither of the converse directions holds. For example, the identity operator on $C^{b}(X)$ is $\beta_{0}-\beta_{0}$ continuous but not $\beta_{0}-\beta_{a}$ continuous in any case where $M_{t} \neq M_{\tau}$ or when there exists a weak ${ }^{*}$ compact subset of $M_{t}^{+}$which is not tight since in both cases $\beta_{0} \neq \beta_{\tau}$ by 5.8 in [9]. Also if $\mu \in M_{\tau}^{+} \backslash M_{t}$ then $T(f)=\mu(f) 1$ is $\beta_{\alpha}-\beta_{0}$ continuous on $C^{b}(X)$ into $C^{b}(X)$ but it is not $\beta_{0}-\beta_{0}$ continuous.

Let us denote $\left\{T \in L^{+}(A, B): T\left(1_{A}\right)=1_{B}\right\}$ by $C$ where $1_{A}$ and $1_{B}$ are the identity functions in $A, B$ respectively.

9. Proposition. Let $T \in C$. Then the following are equivalent. 
(1) $T$ is an extreme point of $C$.

(2) $T$ is an algebraic homomorphism.

(3) $T$ is a lattice homomorphism.

(4) $T^{*}\left(Y_{B}\right) \subset X_{A}$.

Proof. Let $\bar{T}$ be the extension of $T$ from $L^{+}(A, B)$ to $L^{+}\left(C\left(X_{A}\right), C\left(Y_{B}\right)\right)$ given by $\bar{T}(\bar{f})=\overline{T(f)}$. Since the extension of $f$ to $\bar{f}$ from $A$ to $C\left(X_{A}\right)$ and the extension $g$ to $\bar{g}$ from $B$ to $C\left(Y_{B}\right)$ are Banach lattice isomorphisms, we see that $T$ has one of the properties (a)-(c) above if and only if $\bar{T}$ has the same properties. Hence, the result follows from Ellis [2, Theorem 1] since $T^{*}\left(Y_{B}\right)=\bar{T}^{*}\left(Y_{B}\right)$.

10. Proposition. Let $T \in C$. Then $T$ is an extreme point of $C$ and $\tau$ additive iff there exists a continuous function $\phi$ on $\left(Y, \tau_{B}\right)$ into $\left(X, \tau_{A}\right)$ such that $T f(y)=f(\phi(y))$.

Proof. Let $T$ be an extreme point of $C$ and let $T$ be $r$-additive. Then for any $y \in Y$, we have that $\delta_{y} \in Y_{B}$ is $T$-additive by 6 . Hence, $\delta_{y} \in M_{\tau}(z(B))$ so that $T^{*} \delta_{y} \in M_{\tau}(z(A))$ by 7 . However, $T^{*} \delta_{y} \in X_{A}$ by 9 so that $T^{*} \delta_{y}=\delta_{x_{y}}$ for some $x_{y} \in X$ by 6 . Since $A$ separates the points of $X, \phi(y)=X_{y}$ is a well-defined function on $Y$ into $X$ which is clearly $\tau_{A}{ }^{-\tau}{ }_{B}$ continuous.

Conversely, let such a $\phi$ exist. Then since $T^{*}$ is weak ${ }^{*}$ continuous and $\left\{\delta_{y}: y \in Y\right\}$ is weak ${ }^{*}$-dense in $Y_{B}$, we see that $T^{*}\left(Y_{B}\right) \subset \overline{\left\{\delta_{x}: x \in X\right\}}$ $=X_{A}$. Thus by $9, T$ is an extreme point of $C$. If $f_{\gamma} \downarrow \theta$ in $A$ then $\left\{T f_{\gamma}\right\}$ is decreasing in $B$ since $T$ is positive. Since $\lim T f_{\gamma}(y)=\lim f(\phi(y))=0$, it follows that $T$ is $r$-additive.

From this we obtain the following result which extends Mosiman and Wheeler's result $[8,3.1]$.

11. Corollary. If $T$ is an extreme point of $C$, then the following are equivalent.

(a) $T$ is tight.

(b) $T$ is $\tau$-additive.

(c) $T^{*}\left\{\delta_{y}: y \in Y\right\} \subset\left\{\delta_{x}: x \in X\right\}$.

Also if $T$ satisfies any of the above then $T$ is a-additive.

Proof. (b) and (c) are equivalent by 10 and 7.

Since $T$ is an extreme point of $C, T^{*}\left(Y_{B}\right) \subset X_{A}$; hence, (c) follows from (a) because $\left\{\delta_{y}: y \in Y\right\}=Y_{B} \cap M_{t}(z(B))$ and $\left\{\delta_{x}: x \in X\right\}=X_{A} \cap M_{t}(z(A))$ by 6 .

Let $\left\{f_{\gamma}\right\}$ be a norm bounded net of functions in $A$ which converges uniformly to $\theta$ on compact subsets of $X$. Let $\epsilon>0$ and let $K$ be a compact 
subset of $Y$. Then by the weak ${ }^{*}$ continuity of $T^{*}$ and (c), it is easily seen that $K^{\prime}=\left\{x \in X: \delta_{x}=T^{*} \delta_{y}\right.$ for some $\left.y \in K\right\}$ is a compact subset of $X$. Since $T$ is norm continuous, $\left\{T f_{\gamma}\right\}$ is norm bounded in $B$, and $\gamma_{0}$ chosen such that $\left|f_{\gamma}(x)\right|<\epsilon$ for all $x \in K^{\prime}$ when $\gamma \geq \gamma_{0}$ implies that $\left|T f_{\gamma}(y)\right|<\epsilon$ for all $y \epsilon$ $K$ whenever $\gamma \geq \gamma_{0}$. Hence, $T$ is tight so (c) $\Rightarrow(a)$.

Finally, if (a), (b) or (c) holds, then, as in 10, it is easily seen that $T$ is $a$-additive.

We mention that $T$ can be $\beta_{a}-\beta_{a}$ continuous but not $\beta-\beta$ continuous even if $T$ is an extreme point of $C$. For example, let $X=Y=(0, \Omega)$ for $\Omega$ the first uncountable ordinal and $(0, \Omega)$ is equipped with its order topology. Let $\delta_{0}$ be a $\sigma$-additive linear functional given by $\delta_{0}(f)=f\left(x_{f}\right)$ where $x_{f} \epsilon$ $(0, \Omega)$ satisfies $f(y)=f\left(x_{f}\right)$ for all $y \geq x_{f}$. Then $\delta_{0}$ is not $\tau$-additive. Thus, if we define $T$ by $T f=\delta_{0}(f) 1$, then $T$ is an extreme point of $C$ since it is an algebraic homomorphism, but $T$ is $\sigma$-additive and not $\tau$-additive.

Finally we point out that we can determine when a continuous $\phi$ from $Y$ to $X$ can be extended to $Y_{B}$, which could be $\beta Y$ for example.

12. Proposition. Let $\phi$ be a continuous function on $Y$ into $X$. Then $\phi$ has a continuous extension to $Y_{B}$ if and only if $T \in C$ defined by $T f(y)$ $=f(\phi(y))$ is $\beta_{\tau}-\|\cdot\|$ continuous.

Proof. Let $T$ be $\beta_{\tau}-\|\cdot\|$ continuous. Let $f_{\gamma} \downarrow \theta$ on $X$. Then if $W$ is a $\beta_{\tau}$-neighborhood of $\theta$, there is a weak ${ }^{*}$ compact subset $V \subset M_{\tau}^{+}$such that $V^{\circ} C W$ by 3. Then $\left\langle f_{\gamma}, \phi\right\rangle$ converges uniformly to zero for all $\phi \in V$ by Dini's theorem, so there is a $\gamma_{0}$ such that $\gamma \geq \gamma_{0}$ implies $f_{\gamma} \in V^{\circ} \mathrm{CW}$. Hence, $\left\{f_{\gamma}\right\}$ converges to $\theta$ for the $\beta_{\tau}$ topology. Thus, $\left\{T f_{\gamma}\right\}$ converges to $\theta$ uniformly on $Y$. If $\delta_{p} \in Y_{B}$, then $\left\{\delta_{p}\left(T f_{\gamma}\right)\right\}$ converges to zero, so it follows that $T^{*} \delta_{p}$ is $r$-additive. Consequently, $T^{*}\left(Y_{B}\right) \subset\left\{\delta_{x}: x \in X\right\}$ and we extend $\phi$ continuously to $Y_{B}$ by $\phi(p)=x$ for $T^{*}\left(\delta_{p}\right)=\delta_{x}$.

Conversely let $T$ be defined by $T f(y)=f(\phi(y))$ where $\phi$ has a continuous extension from $Y$ to $Y_{B}$. Let $\left\{f_{\gamma}\right\} \beta_{\tau}$-converge to $\theta$. Then we may assume $\left\{f_{\gamma}\right\}$ is norm bounded by 2. Since $T^{*}\left(Y_{B}\right)=\left\{\delta_{x}: x=\phi(p)\right.$ for $p \epsilon$ $\left.Y_{B}\right\}$, and $T^{*}\left(Y_{B}\right)$ is compact in $X_{A},\left\{f_{\gamma}\right\}$ converges uniformly to $\theta$ on $T^{*}\left(Y_{B}\right)$ (see [9, Theorem 5.9]). Consequently $\left\{T f_{\gamma}\right\}$ converges uniformly to $\theta$ in $Y$ and it follows that $T$ is $\beta_{\tau}-\|\cdot\|$ continuous.

\section{REFERENCES}

1. F. F. Bonsall, J. Lindenstrauss and R. R. Phelps, Extreme positive operators on algebras of functions, Math. Scand. 18 (1966), 161-182. MR 35 \#759.

2. A. J. Ellis, Extreme positive operators, Quart. J. Math. Oxford Ser. (2) 15 (1964), 342-344. MR $30 \# 4157$. 
3. J. Hoffman-J 6 rgensen, A generalization of the strict topology, Math. Scand. 30 (1972), 313-323.

4. R. B. Kirk, Algebras of bounded real-valued functions. I, II, Nederl. Akad. Wetensch. Proc. Ser. A 75=Indag. Math. 34 (1972), 443-463.

5. R. B. Kirk and J. A. Crenshaw, A generalized topological measure theory (to appear).

6. J. D. Knowles, Measures on topological spaces, Proc. London Math. Soc. (3) 17 (1967), 139-156. MR 34 \#4441.

7. S. E. Mosiman, Strict topologies and the ordered vector space $C(X)$ (to appear).

8. S. E. Mosiman and R. F. Wheeler, The strict topology in a completely regular setting: relations to topological measure theory, Canad. J. Math. 24 (1972), 873-890.

9. F. D. Sentilles, Bounded continuous functions on a completely regular space, Trans. Amer. Math. Soc. 168 (1972), 311-336. MR 45 \#133.

10. V. S. Varadarajan, Measures on topological spaces, Mat. Sb. 55 (97) (1961), 35-100; English transl., Amer. Math. Soc. Transl. (2) 48 (1965), 161-228. MR 26 \#6342.

DEPARTMENT OF MATHEMATICS, SOUTHERN ILLINOIS UNIVERSITY, CARBONDALE, ILLINOIS 62901

Current address: 1058 Loma Verde, Palo Alto, California 94303 